\title{
Éditorial: De la paternité des articles
}

\begin{abstract}
Note du Rédacteur en chef: Les questions portant sur la création intellectuelle sont celles qui me sont des plus souvent posées par les érudits débutants. Même les érudits supérieurs discutent souvent de cette question. Ces discussions révèlent que les opinions sont divergentes et au sujet de la paternité des cuvres et sous quelles conditions celle-ci devrait être affectée. La création intellectuelle est un sujet important autant pour les rédacteurs de revues savantes que pour les auteurs. Lorsqu'il était Rédacteur en chef de cette revue, Victor W. Marshall, a écrit un éditorial au sujet de la création intellectuelle afin d'éclaircir les décisions prisent à ce sujet (La revue canadienne du vieillissement, (1986), 5(3), 148-151). Cet éditorial demeure utile et de mise même s'il date déjà de sept ans. J'encouragerais les lecteurs qui se retrouvent en désaccord avec les affirmations ou les lignes directrices de cet éditorial ou qui ont des questions au sujet de la création intellectuelle de soumettre une tribune libre.
\end{abstract}

L'activité scientifique des chercheurs est attestée avant tout par les articles publiés dans les revues savantes, notamment dans les revues dotées d'un comité de lecture. La publication d'un article honore son auteur et lui vaut la considération des ses collègues. Pourtant, malgré 1'importance accordée à la publication des communications scientifiques dans le processus de reconnaissance du mérite professionnel (prestige, avancement, etc.), ou peut-être justement à cause de celle-ci, les règles gouvernant 1 'attribution de la paternité intellectuelle ne sont pas toujours parfaitement claires. Dans le cas des rédigés en collaboration, la question de savoir si tel nom doit figurer parmi la liste des auteurs, et dans quel ordre les divers noms doivent apparaître, suscite souvent des débats et des inquiétudes. Pour ma part, je suis persuadé que les erreurs d'omission et de commission sont fréquentes. Or, s'il s'agit certes d'éthique, ce n'est pourtant pas seulement 1'éthique qui est en cause, mais bien la fiabilité du processus scientifique et le contrôle de la qualité des écrits.

Le «groupe de Vancouver » qui réunit des rédacteurs attachés au domaine biomédical et à qui on doit un des protocoles reconnus par notre revue, le " protocole de Vancouver », a énoncé des propositions directrices en vue de définir la qualité d'auteur et de normaliser la référence aux sources (International Committee of Medical Journal Editors, 1985). On trouve aussi des considérations utiles sur ces questions dans les ouvrages de Day (1983) et de Diener et Crandall (1978). Ces auteurs proposent des normes qui me semblent raisonnables et dont on aurait intérêt à tenir compte tant au moment de la présentation d'une communication qu'au stade de 1 'organisation de la recher- che, chaque fois que 1'on envisage un travail collectif. Voici donc un bref résumé de ces normes et propositions.

Le principe fondamental formulé par le groupe de Vancouver comporte trois volets et s'énonce ainsi : «La participation de chaque auteur doit être suffisante pour que celui-ci assume publiquement la responsabilité du contenu de 1'étude. Cette participation doit comprendre: (a) ou bien la conception, ou bien 1'analyse et 1 'interprétation des données, ou les deux à la fois ; (b) la première rédaction de 1'article, ou une revision touchant les points les plus importants de son contenu intellectuel ; et (c) 1'approbation de la version destinée à la publication » (c'est nous qui traduisons). Selon ces critères, on peut considérer comme auteur toute personne à qui 1'on doit la conception d'une étude, même si elle n'a pas analysé ellemême les données recueillies ni rédigé [elle-même] le premier jet, à condition toutefois que sa contribution intellectuelle à la rédaction du manuscrit soit importante et qu'elle ait donné son accord à la publication du manuscrit définitif. On peut également considérer comme auteur tout chercheur qui manipule les données recueillies au cours d'une étude dont il n'est pas le concepteur, à condition qu'il participe activement à 1 'analyse ou à 1'interprétation de ces données, qu'il apporte sa contribution intellectuelle à 1'élaboration de 1'article et qu'il en approuve la version définitive.

Le groupe de Vancouver précise ensuite ce qui ne donne pas le droit de revendiquer la paternité d'une œuvre : « Le fait de participer uniquement à la collecte des données ne donne pas droit au titre d'auteur» (c'est nous qui traduisons). En vertu de ce principe, ni 1'enquêteur qui recueille des données en vue d'une étude sociologique, par exemple, ni le médecin qui met des patients à la disposition des chercheurs ne sont autorisé à se considérer comme des auteurs. Autrement dit, le seul fait de recueillir et de réunir des données ou de présenter les répondants aux chercheurs ne justifie pas 1'attribution du titre d'auteur. Si j'insiste sur ce dernier point, c'est que j'ai eu connaissance de directeurs de clinique qui réclamaient d'être cités comme co-auteurs pour les motifs mentionnés cidessus. Or ces motifs sont insuffisants. Par ailleurs, il est bien entendu que si le directeur d'une clinique a collaboré à une recherche en persuadant ses clients de participer à une étude et qu'il a également fourni une contribution intellectuelle, $\mathrm{il}$ a le droit de se considérer comme co-auteur. Diener et Crandall (1978, p. 144) soulignent en outre que «... le prêt d'équipement scientifique et 1'application de cet équipement aux sujets justifient une mention dans les notes de bas de page 
mais non le titre de co-auteur » (c'est nous qui traduisons). Cela signifie que les directeurs de laboratoires ne sauraient réclamer le titre de co-auteur simplement parce que la recherche a été effectuée dans "leur labo ».

Le déni du titre d'auteur aux auxiliaires chargés de la collecte des données n'a rien à voir avec la versement d'une rémunération. Auxiliaires et assistants rémunérés peuvent se considérer comme des coauteurs au même titre que les auxiliaires et assistants bénévoles, à condition toutefois de contribuer à 1'analyse des données. La contribution intellectuelle constitue en effet le critère décisif qui permet de justifier le titre d'auteur, et ce critère signifie que quiconque réclame la mention de co-auteur est en mesure de prendre la parole dans une réunion savante et d'en défendre 1'analyse.

Idéalement, 1'ordre de présentation des noms d'auteur devrait correspondre à 1'importance relative des contributions. Les auteurs dont les noms apparaissent en position initiale bénéficient en effet d'une degré de considération supérieur à celui qui est accordé aux auteurs nommés ensuite. À moins d'avis contraire, on suppose que 1 'auteur nommé en premier lieu est celui qui a fourni la contribution la plus importante. Dans les citations rapides, il n'est pas rare que seul le nom du premier auteur apparaisse, les autres étant englobés dans la mention «et collaborateurs ». Quand tous collaborent à la recherche dans la même mesure, les auteurs peuvent choisir la solution du roulement, qui consiste à placer chaque chercheur en position initiale à tour de rôle à mesure des publications ; une autre solution consiste à adopter 1'ordre alphabétique en indiquant dans une note de bas de page que toutes les contributions sont à peu près égales. Ni le rang professionnel des divers auteurs, ni la qualité de professeur et d'étudiant, ni la différence des sexes ne doivent entrer en ligne de compte au moment de décider de 1'attribution de la paternité des ouvrages et des articles ou de déterminer la position respective des noms dans la liste des auteurs; il ne convient pas non plus de considérer 1 'inclusion d'un nom comme une récompense offerte à ceux qui ont beaucoup travaillé (Diener and Crandall, 1978, p. 164-165), car on ne saurait établir une corrélation entre la durée des prestations de travail et le qualité du travail intellectuel.

Il est souhaitable de convenir de 1'attribution du titre d'auteur et de 1 'ordre de présentation des noms avant de rédiger 1'article, voire avant d'entreprendre la recherche. Dans la pratique, cependant, il arrive souvent que la part de travail intellectuel fournie par cha- cun varie au cours de 1'étude et même au cours de la rédaction d'un rapport de recherche. Les collaborateurs devraient donc s'entendre au préalable, surtout lorsqu'ils se trouvent en présence d'une différence de rang, comme lors de la collaboration professeur-étudiant, par exemple, et peut-être prévoir 1'appel à un médiateur en cas de désaccord.

La justice la plus élémentaire exige que 1 'on reconnaise 1 'apport des collaborateurs qui ne peuvent prétendre à la qualité d'auteur. Dans notre revue, une note au bas de la première de page leur est consacrée, en même temps que les remerciements aux organismes qui ont financé la recherche. La mention de 1'aide fournie pas les enquêteurs, secrétaires et techniciens devrait inclure ceux qui ont prodiqué leurs "conseils", consenti des "communications verbales", et bien voulu « relire le manuscrit ».

Ne pas mentionner un collaborateur qui mérite le titre d'auteur constitue une exploitation illicite de la personne humaine; attribuer injustement ce titre à qui n'y a pas droit équivaut à saper le système de reconnaissance du mérite professionnel. Enfin j'estime que 1'attribution inconsidérée de la paternité intellectuelle constitue un problème qui se généralise et qui menace 1'intégrité du processus scientifique. C'est également une injustice, puisque cela diminue aux yeux des lecteurs 1'apport des collaborateurs légitimes.

L'attribution de la paternité d'un article est donc une question complexe qui tire à conséquence. Le groupe de Vancouver a déclaré que «les directeurs peuvent exiger des auteurs la justification du titre d'auteur » (c'est nous qui traduisons). Le comité de rédaction de La revue canadienne $d u$ vieillissement se réserve le droit d'exiger une telle justification le cas échéant et de refuser de publier les articles, même valables, dont les auteurs refuseraient de se conformer à cette exigence.

Victor W. Marshall

Université de North Carolina à Chapel Hill (lorsque cet éditorial a été écrit, le professeur Marshall était à l'Université de Toronto).

\section{Reférences}

Day, Robert A. (1983). How to Write and publish a scientific paper. (2nd ed.). Philadelphia: ISI Press.

Diener, Edward \& Crandall, Rick (1978). Ethics in social and behavioral research. Chicago: The University of Chicago Press.

International Committee of Medical Journal Editors. (1985). Guidelines on authorship. British Medical Journal 291(6497), 722. 